\title{
TAGUNG
}

\section{Wirtschaftspolitischer Reformbedarf in Europa}

\author{
Sarah Jamil und Alexander Lenger*
}

Deutschland und andere westeuropäische Länder weisen noch immer einen hohen Reformbedarf ihrer staatlichen Sicherungssysteme, Arbeitsmarktinstitutionen, Steuersysteme oder föderalen Ordnungen auf. Zudem zeigt sich am Umgang mit den jungen Marktwirtschaften in Osteuropa, dass westliche Länder beträchtliche Schwierigkeiten haben, angemessen auf neue Situationen und Herausforderungen $\mathrm{zu}$ reagieren. Trotzdem finden Lösungsvorschläge von Wirtschaftswissenschaftlern und -wissenschaftlerinnen in der Praxis nur selten Anwendung. Ursachen hierfür sind oft die fehlende Berücksichtigung des politischen Prozesses und die alleinige Konzentration auf wirtschaftliche Faktoren. Thema der Tagung war deswegen, wie der politische Prozess besser in die ökonomische Analyse integriert werden kann. Hierzu waren neben Referentinnen und Referenten aus den Wirtschaftswissenschaften insbesondere auch Vortragende aus der Psychologie eingeladen.

Eröffnet wurde die Tagung von Bernhard Neumärker und Friedrich Heinemann mit einigen einführenden Bemerkungen zur Ausrichtung der gastgebenden Arbeitskreise und dem Hinweis auf die Stagnation von Reformprozessen in Europa. Die Vorträge waren in sechs Panels unterteilt, welche in jeweils drei Parallelsitzungen stattfanden.

Internationale Dimension wirtschaftspolitischer Reformen

Im ersten Beitrag argumentierte Alexander Lenger, dass die Wahlentscheidung zwischen Schocktherapie und gradueller Reform die

\section{Ökonomik, Politische Ökonomie und Psychologie staatlicher Reformen in Europa}

Frühjahrstagung 2008 des Arbeitskreises Politische Ökonomie in Zusammenarbeit mit dem Arbeitskreis Europäische Integration, dem

Walter Eucken Institut Freiburg und dem Zentrum für Europäische Wirtschaftsforschung Mannheim

Mit Unterstützung der Europäischen Kommission

Freiburg, 10./11. April 2008

Wissenschaftliche Leitung, Begrüßung und Einführung

Prof. Dr. K. J. Bernhard NEUMÄRKER, Universität Freiburg; Walter Eucken Institut Freiburg Dr. Friedrich HEINEMANN, Zentrum für Europäische Wirtschaftsforschung Mannheim

Quantifizierung von Reformen und internationale Vergleiche

Shock Therapy versus Gradualism? Transition

Processes in Eastern Europe Reviewed

Alexander LENGER, Universität Freiburg

Der Bertelsmann Reform Index - ein neues Instrument zur Messung von Reformen Oliver HEILWAGEN, Bertelsmann Stiftung

Yardstick Competition through the Open Method of Coordination - the Example of Health Care Reforms in the EU

PD Dr. Martina ECKARDT, Prof. Dr. Stefan OKRUCH, Andrássy-Universität Budapest

Reformen fiskalischer und föderaler Regelwerke

Die Reform des Stabilitäts- und Wachstumspaktes: Missglücktes Konstrukt und/oder Politikversagen Prof. Dr. André SCHMIDT, European Business School, Oestrich-Winkel

* Dipl.-Vw. Sarah Jamil, Institut für Allgemeine Wirtschaftsforschung, Abteilung für Wirtschaftspolitik, AlbertLudwigs-Universität Freiburg.

Dipl.-Vw. Alexander Lenger, M.A., Institut für Allgemeine Wirtschaftsforschung, Abteilung für Wirtschaftspolitik, Albert-Ludwigs-Universität Freiburg. 
unterschiedlichen Transformationserfolge in Zentral- und Osteuropa alleine nicht erklären könne. Während Länder in Zentral- und Osteuropa und den Baltischen Staaten bereits heute erfolgreiche marktwirtschaftliche Ordnungen aufwiesen, stehe in vielen Ländern der ehemaligen Sowjetunion diese Entwicklung noch aus. Als vermeintliche Ursache hierfür gelte vielen Ökonomen die Wahl der Reformstrategie, denn während in den meisten erfolgreichen Ländern die Transformation als Schocktherapie organisiert wurde, wählten viele weniger erfolgreiche Länder einen graduellen Transformationsprozess. Hierbei - so die zentrale These von Lenger - handele es sich aber um eine Scheinkorrelation. Denn der wirtschaftliche Erfolg sei nicht das Resultat einer bestimmten Reformstrategie, sondern stelle das Ergebnis einer besseren kulturellen Anpassungsfähigkeit an marktwirtschaftliche Funktionsprinzipien dar. Empirische Befunde zeigten, dass sowohl kulturelle Ähnlichkeiten $\mathrm{zu}$ westlichen Institutionen als auch die ökonomische Ausgangssituation der betroffenen Länder ihren späteren wirtschaftlichen Erfolg beeinflussten. Lenger sprach sich deswegen dafür aus, die Transformationsprozesse in Zentral- und Osteuropa aus der Perspektive einer kulturellen Ökonomik zu untersuchen.

Einen neuen Index zur Messung von Reformen stellte Oliver Heilwagen vor. Die Besucher seines Vortrags hatten das Privileg, dass ihnen als Erste die Ergebnisse des Bertelsmann-Projektes präsentiert wurden. Der Bertelsmann Reform Index analysiert den Reformbedarf und die Reformfähigkeit von 30 OECD-Staaten. Ziel des Bertelsmann Reform Index sei die vergleichende Bewertung von Regierungsleistung und Reformfähigkeit der konsolidierten Demokratien in der OECD mit Blick auf zentrale Zukunftsherausforderungen. Zentraler Befund der Studie sei, dass die skandinavischen Länder eine höhere Konsensfähigkeit aufwiesen. Die Ergebnisse der Untersuchung werden im Oktober 2008 veröffentlicht und die Daten sollen der interessierten Forschungsgemeinde mittels einer öffentlichen Internetplattform zugänglich gemacht werden.
Das Verschwinden von Budgetdefiziten - eine Erfolgsgeschichte des Wachstums- und Stabilitätspakts? Das Beispiel Deutschland Prof. Hubert HIEKE, Ph.D., Ryszard Lazarski Universität Warschau

Reform und Status quo im deutschen Föderalismus aus institutionenökonomischer Perspektive Jan VOßWINKEL, Ruhr-Universität Bochum

Patriotismus, Konstitutionenökonomie und Makroökonomie der Reformen

„Wir sind Deutschland“ - Der Einfluss des Bewusstseins für die Zugehörigkeit zu einer deutschen Gemeinschaft auf die Akzeptanz von Reformen

Rebecca DÖRFLER, Universität München

Konstitutionelle Reform und konstitutionelle Logik von Reformen

Prof. Dr. K. J. Bernhard NEUMÄRKER, Universität Freiburg; Walter Eucken Institut Freiburg

Flexicurity Capitalism

Prof. Dr. Peter FLASCHEL, Universität Bielefeld

Reformen internationaler Organisationen Reform und Lernen internationaler Institutionen - die Bank für Internationalen Zahlungsausgleich (BIZ)

PD Dr. Elke MUCHLINSKI, Freie Universität Berlin

Politische Ökonomik der Reform des Europäischen Gerichtshofs

Andreas KOENIG, Universität Hohenheim

Who is Afraid of an EU Tax and Why?

Dr. Friedrich HEINEMANN, Zentrum für Europäische Wirtschaftsforschung Mannheim

Keynote: Die Psychologie wirtschaftspolitischer Reformen - ein Überblick

Prof. Dr. Detlef FETCHENHAUER, Universität Köln

\section{Glück, Vertrauen, Angst und Reformen}

Angst und Reformen - Welche politischen Implikationen ergeben sich aus der Terror Management Theorie für die Akzeptanz von Reformen? Luitgard REIß, Universität München

Die glückliche Variante des Kapitalismus Dr. Stefan BERGHEIM, Deutsche Bank Research

Vertrauen und Reformen

Dr. Friedrich HEINEMANN, Zentrum für Europäische Wirtschaftsforschung Mannheim 
Abschließend diskutierten Martina Eckardt und Stefan Okruch am Beispiel der Gesundheitsreform die Bedeutung der offenen Methode der Koordinierung (OMK) für einen Systemwettbewerb innerhalb der Europäischen Union. Die offene Methode der Koordinierung habe sich seit den 1990er Jahren zu einem gut funktionierenden Steuerungsinstrument in der Gesundheitspolitik entwickelt. Mithilfe der evolutorischen Theorie der Wirtschaftspolitik zeigten Eckardt und Okruch, dass dieser seit 2004 im Gesundheitsbereich regelmäßig angewendete ,Good Practice'-Ansatz in den Mitgliedstaaten zu einem erheblichen Politiklernen durch den Austausch von Informationen innerhalb der Mitgliedstaaten geführt habe.

Reform des Stabilitäts- und Wachstumspaktes sowie Reform und Status quo im deutschen Föderalismus

Den Auftakt zur Auseinandersetzung mit fiskalischen und föderalen Regelwerken machte André Schmidt. Beleuchtet wurde der Stabilitäts- und Wachstumspakt (SWP) hinsichtlich seiner ökonomischen Legitimation und zu empfehlender Reformansätze. Aus konstitutionenökonomischer Perspektive kritisierte Schmidt die hohe Instabilität des SWP, insbesondere die Möglichkeit, durch Abweichen von der eigenen Position Verbesserungen zu realisieren. Ein weiterer Kritikpunkt war die fortwährende Aushöhlung des SWP, was zu zahlreichen VerstöBen gegen die Defizitkriterien führe. $\mathrm{Zu}$ den von Schmidt empfohlenen Reformkriterien zählen die Aufgabe der Drei-Prozent-Grenze, eine Orientierung an Tragfähigkeitskriterien der Verschuldung sowie die Berücksichtigung politökonomischer Aspekte und Auswirkungen auf bestehende Anreizmechanismen.

Auch im zweiten Vortrag wurde der SWP thematisiert, diesmal durch Hubert Hieke. Anhand empirischer Daten konnte gezeigt werden, dass die Länder, die den Stabilitätspakt erfüllen, mit Preisstabilitätsproblemen zu kämpfen haben und gleichzeitig hohe Wachstumsraten aufweisen. Hingegen wiesen die Länder, die gegen den SWP verstoßen haben, häufig eine hohe Preisstabilität auf. Hieraus
Reformprobleme öffentlicher Deregulierung und des Gesundheitswesens

The Miscellaneous Desirability of Public-Private Partnerships and an Approach to Design an Appropriate Constitution

Sarah JAMIL, Universität Freiburg

Reagieren Opfersensitive stärker auf Reformen? Unterschiede aufgezeigt am Beispiel der Gesundheitsreform und der Einführung von Studiengebühren

Dr. Eva TRAUT-MATTAUSCH, Universität München

Keynote: Rolle rückwärts bei der Agenda 2010 - kehrt der Reformstau zurück?

Oswald METZGER, Bad Schussenried

schlussfolgerte Hieke, dass sektorale Finanzierungsströme nicht außer Acht gelassen werden sollten. Die Bedeutung der privaten Ersparnis und deren Wichtigkeit für Investitionen wurden deutlich hervorgehoben. Darauf aufbauend kritisierte Hieke, dass die Europäische Zentralbank zu stark auf das Drei-Prozent-Kriterium fokussiert sei und dabei die Länder vernachlässige, die sich durch eine hohe private Ersparnis auszeichneten.

Der abschließende Beitrag kam von Jan Voßwinkel. In seinem Vortrag hob Voßwinkel die Bedeutung von Verfassungen mit dauerhafter Regel und die Notwendigkeit eines quasipermanenten Ordnungsrahmens hervor. Anhand der Entwicklung des Föderalismus in der Bundesrepublik wies er darauf hin, dass sich der Wandel des Föderalismus in einem Wechsel von Phasen der intentional herbeigeführten Reform der konstitutionellen Struktur und innerperiodischen Anpassungen vollziehe. Abschließend wurden die Ergebnisse der Föderalismuskommission II und die Rolle des Status quo im Hinblick auf Reformen dargestellt.

Reformen und Patriotismus aus psychologischer und konstitutionenökonomischer Perspektive

Rebecca Dörfler untersuchte in ihrem zweiteiligen Beitrag den Zusammenhang zwischen 
nationalem Bewusstsein und der Akzeptanz von Reformen. Im ersten Teil führte sie hierzu das ,Common Ingroup Identity Model ${ }^{\circ}$ ein, mit welchem sie im zweiten Teil prüfte, ob das Zugehörigkeitsbewusstsein zu einer übergeordneten Kategorie - im Fall von Reformgewinnern und Reformverlierern zur inklusiven Gruppe ,deutsche Bürger - auch für politische beziehungsweise wirtschaftliche Kontexte akzeptanzsteigernde Wirkung hat. Anhand einer Untersuchung des Solidaritätszuschlags konnte gezeigt werden, dass sich Gruppenidentität im politischen Reformkontext nutzbar machen lässt, da sie die Zustimmungsfähigkeit zu Reformen erhöht. So werde zum Beispiel der Solidaritätszuschlag eher akzeptiert, wenn ein nationales Zugehörigkeitsgefühl besteht.

Bernhard Neumärker widmete seinen Beitrag dem Thema,konstitutionelle Reform und konstitutionelle Logik von Reformen'. Hierzu grenzte er zunächst die spontane Änderung von Normen von der geplanten Gestaltung durch bewusste öffentliche Entscheidung ab und verglich die Status quo-Bezogenheit verfassungsmäßiger Änderungen, bei der die Gesamtheit aller bestehenden Regeln und deren Wirkungen bei einer Regelreform zu beachten seien, mit dem Verfassungsdesign, bei dem als relevanter Ansatzpunkt die Anarchie gewählt werde. Darauf aufbauend präsentierte Neumärker einen Überblick über die positive Theorie konstitutioneller Reformen und die konstitutionelle Logik wirtschaftspolitischer Reformen. Abschließend wurden die Anforderungen an einen Verfassungsmechanismus dargestellt, der die unvollständigen postkonstitutionellen Informationsdefizite der Bürger berücksichtigt und auf die zwei zentralen Funktionen konstitutioneller Regeln für postkonstitutionelle Reformaktivitäten verwiesen: Erstens beschränkten sie die Macht öffentlicher Entscheidungsträger bei Reformaktivitäten und zweitens dienten sie als Anreizinstrument zur Signalisierung politischer Glaubwürdigkeit und zeitkonsistenten Verhaltens.
Peter Flaschel präsentierte das Konzept des ,Flexicurity Capitalism‘. Zunächst stellte er ein grundlegendes makrodynamisches Rahmenwerk mit verschiedenen Formen zyklischen Wachstums und sozialer Reproduktion vor. Das Konzept zeige, dass eine Kombination aufbauend auf Ideen von Marx, Keynes und Schumpeter eine echte Alternative zum neoklassischen Wettbewerbsmodell darstelle. Der entscheidende Grund, so Flaschel, der für eine Hinwendung zum Flexicurity Modell spreche, resultiere aus der Tatsache, dass alternative kapitalistische Reproduktionsschemen keine sozialen Strukturen böten, die langfristig mit einer gebildeten und demokratischen Gesellschaft kompatibel sind. Massenarbeitslosigkeit untergrabe die soziale Kohäsion und führe zu sozialer Segmentation - ein Problem, welches die Mainstreamökonomik in ihrem Modelldenken nicht integrieren könne.

\section{Reformierbarkeit internationaler Organisa- tionen}

Elke Muchlinski referierte zunächst exemplarisch anhand der Bank für Internationalen Zahlungsausgleich über die Notwendigkeit von institutionellem Lernen und Reformen in Organisationen. Anknüpfend an die moderne Theorie der Institutionen thematisierte Muchlinski relevante Lernfaktoren für Institutionen, wie die Kontextabhängigkeit von Lernprozessen, die kulturelle Eingebundenheit von Institutionen sowie die Annäherung der Institutionenökonomik an die Kognitionswissenschaft und die evolutionäre Lerntheorie. Muchlinski gelang es, in ihrem Vortrag zu zeigen, dass eine Erweiterung der theoretischen Perspektive um diese Elemente grundlegend geeignet sein kann, die institutionelle Lernfähigkeit der Bank für Internationalen Zahlungsausgleich zu erklären.

Mit der Reform des Europäischen Gerichtshofs (EuGH) aus politökonomischer Perspektive beschäftigte sich Andreas Koenig. Er argumentierte, dass sowohl theoretische Argumente als auch empirische Evidenz gegen 
das häufig prognostizierte Konkurrenzverhältnis zwischen EuGH und Mitgliedstaaten sprächen. Vielmehr belegten die abnehmende Anzahl zentralisierungsfreudiger EuGHUrteile bereits den impliziten Wandel, welchem der EuGH durch institutionellen Wandel unterliege. So zeige sich, dass eine explizite Reform aus Sicht der nationalen Regierungen nicht mehr unbedingt erforderlich sei, da der konstitutionelle Wandel der Europäischen Union auch nicht vor der - formal unabhängigen - Judikative halt gemacht habe.

Friedrich Heinemann präsentierte dem Auditorium im Anschluss eine empirische Untersuchung zur Akzeptanz einer möglichen EUSteuer unter Abgeordneten des Europäischen Parlaments. Vor dem Hintergrund der Nettozahlerdebatte wurde die Frage diskutiert, ob sich die Europäische Union nicht doch eher über eine EU-Steuer als über Beiträge finanzieren sollte. Dabei ergaben sich drei zentrale Befunde: Erstens erfolge eine Befürwortung einer EU-Steuer entlang ideologischer Präferenzen. Während Grüne und Sozialisten eine solche Steuer tendenziell befürworteten, lehnten Konservative und Liberale sie eher ab. Zweitens überrasche der Befund, dass persönliche Faktoren einen Einfluss ausübten. Je älter und je länger ein Abgeordneter Mitglied des Europäischen Parlaments sei, desto wahrscheinlicher sei ein Sozialisationseffekt dieser Mitgliedschaft. Drittens liege ein länderspezifischer Einfluss vor, da eine steuerfinanzierte Europäische Union von den Abgeordneten der neuen Mitgliedstaaten abgelehnt werde. Insgesamt zeigten die Ergebnisse, dass Mitglieder des Europäischen Parlaments nicht an eine vermeintliche Effektunabhängigkeit der EU-Steuer glauben.

\section{Emotionen und Vertrauen im Reformprozess}

Zunächst beschäftigte sich die Sozialpsychologin Luitgard Reiß mit der Funktion von instinktiven und existenziellen Ängsten im Reformprozess. Das Referat ging der Frage nach, inwieweit Befunde der ,Terror Management Theorie' auf den Reformkontext über- tragen werden können. Aus Sicht dieser Theorie erlebten Menschen, wenn ihnen ihre eigene Sterblichkeit bewusst gemacht werde, existenzielle Angst, der sie begegneten, indem sie ihr kulturelles Weltbild verteidigten und versuchten, ihren Selbstwert zu erhöhen. Im Rahmen des Referats wurden erste Forschungsergebnisse zu Fragen des EU-Grenzschutzes präsentiert. Untersuchungen von Reiß haben gezeigt, dass Menschen mit experimentell induziertem Bewusstsein über die eigene Sterblichkeit einer Grenzöffnung der Europäischen Union negativer gegenüberstehen und eine Verschärfung des EU-Grenzschutzes befürworten. Diese Erkenntnisse belegten die realpolitische Bedeutung von Angst für wirtschaftspolitische Reformen.

Stefan Bergheim betrachtete hingegen die Emotionen Glück und Lebenszufriedenheit. Vor dem Hintergrund, dass fast alle OECDStaaten einen hohen materiellen Wohlstand realisiert haben, stelle sich für diese Gesellschaften die Frage, in welche Richtung sie sich weiterentwickeln wollen. Orientierung, so Bergheim, biete die interdisziplinäre Glücksforschung. Dabei unterschieden sich Vorgehensweise und Ergebnisse der Glücksforschung substanziell von denen der Wohlfahrtsökonomik. Anstatt menschliche Verhaltensweisen nur zu beobachten, ließen Glücksforscher die betroffenen Menschen zu Wort kommen - mit dem Ergebnis, dass Glück oder Lebenszufriedenheit empirisch messbar und somit auch vergleichbar seien. Bergheim präsentierte die Befunde einer empirischen Studie von 22 reichen Ländern, die eine ,glückliche Variante des Kapitalismus" nahelegten. Australien, die Schweiz, Kanada, Großbritannien, die USA, Dänemark, Schweden, Norwegen und die Niederlande sowie in eingeschränkter Form Finnland und Neuseeland hätten ihre Gesellschaften und Institutionen so organisiert, dass die für die Lebenszufriedenheit der Menschen wichtigen Bedingungen gegeben seien. Die Eigenschaften der glücklichen Variante sind unter anderem hohe Beschäftigungsquoten Älterer, große wirtschaftliche Freiheit, niedriger Ar- 
beitsplatzschutz, eine hohe Geburtenrate sowie ein hohes Vertrauen in Mitmenschen.

Mit dem Einfluss von Vertrauen auf wirtschaftspolitische Reformprozesse setzte sich abschließend Friedrich Heinemann ausführlicher auseinander. Dass Vertrauen eine zentrale Rolle für wirtschaftliche Prozesse spiele, sei hinlänglich bekannt: Vertrauen senke Transaktionskosten, es löse Kooperationsprobleme und es erzeuge Glaubwürdigkeit bei Exante-Entscheidungen. Dementsprechend komme Vertrauen eine zentrale Rolle im Reformprozess zu. Allerdings sei diese Funktion noch nicht empirisch untersucht worden. Mithilfe des World Values Survey schließt Heinemann diese Lücke. Seine Untersuchung belege, dass allgemeines Vertrauen die Durchführung von politischen Reformen erleichtere. Sie zeige aber auch, dass Vertrauen in bestimmte Organisationen und Institutionen, wie zum Beispiel in Parlamente, Gewerkschaften, Firmen oder das Rechtssystem, als sehr ambivalent einzustufen sei. Die Ursache hierfür sieht Heinemann in verschiedenen Vertrauenskategorien. Während allgemeines Vertrauen in Mitmenschen auch für einmalige Reformsituationen brauchbar sein könne, scheinen Fragen nach Vertrauen in Organisationen und Institutionen das Reziprozitätsvertrauen zu aktivieren, also nur für Reformen bei mehrfach wiederkehrenden Situationen anwendbar zu sein.

\section{Reformprobleme öffentlicher Deregulierung und des Gesundheitssystems}

Zunächst referierte Sarah Jamil über die verschiedenen Vorteile von ,Public-Private Partnerships ' (PPP) und setzte sich kritisch mit deren Finanzierungsaspekten auseinander. Anhand eines historischen Überblicks über öffentlich-private Partnerschaften arbeitete sie heraus, dass PPP kein neues Konzept seien. Sie hob sowohl die politische Dimension von PPP als auch deren Wirkung auf den öffentlichen Haushalt hervor, um zu zeigen, dass PPP nicht immer die optimale Beschaffungsalternative seien. Mithilfe eines formalen Modells machte sie deutlich, dass es be- stimmte Bedingungen gebe unter denen PPP vorzuziehen sind und andere unter denen herkömmliche öffentliche Bereitstellung zu empfehlen sei. So zeige sich, dass PPP keinesfalls immer als schlecht zu bewerten seien. Ihre Vorteilhaftigkeit, das heißt in erster Linie die kurzfristig geringere Belastung der öffentlichen Haushalte, berge für die Politik die Gefahr einer inflationären Umsetzung dieser Beschaffungsvariante und zwar auch in Fällen in denen öffentliche Bereitstellung vorzuziehen wäre. Den Abschluss bildete die Empfehlung, Verfassungen so zu gestalten, dass Politiker, gerade wegen ihres Informationsvorteils gegenüber Bürgern PPP nur dann umsetzen, wenn es das Regelwerk vorsieht.

Eva Traut-Mattausch referierte anschließend über das Empfinden von Opfersensitiven in Bezug auf Reformen am Beispiel der Gesundheitsreform und der Studiengebühren. Im Vordergrund stand die These, dass es negative Reaktionen auf Reformen gebe, die sich in antisozialem Verhalten äußern könnten. Dies trete insbesondere dann auf, wenn Reformen als illegitime Freiheitsbeschränkung erlebt würden. Die Folge sei eine emotionale Reaktion, die darauf gerichtet sei, diese Einschränkung zu revidieren. Gegenstand der Untersuchung war die Frage, ob es Personen gibt, bei denen diese Sensitivität aufgrund ihrer Persönlichkeit öfter auftritt. Hierzu wurde zunächst analysiert, ob es überhaupt eine Sensibilität für Ungerechtigkeit gibt. Befund der Untersuchung war, dass das Empfinden von Reformen auf die Ungerechtigkeitssensibilität zurückgehe. Nutznießer von Reformen befürworteten eher prosoziales Verhalten, während ,Verlierer' die Reform als illegitime Freiheitsbeschränkung empfänden, was häufig in antisozialem Verhalten kanalisiert werde.

\section{Fazit}

Die Vielzahl der vorgestellten Themen verdeutlichte eindrücklich die Notwendigkeit interdisziplinärer Forschung über Fragen zum Reformbedarf in Europa. Die Vorträge machten deutlich, dass, um den politischen Prozess in die ökonomische Analyse zu integrieren, es 
der nachhaltigen Erweiterung rein ökonomisch orientierter Modelle bedarf. Dabei stellt insbesondere der Dialog zwischen Ökonomie und Psychologie eine vielversprechende Forschungssynthese dar, um zu realitätsnahen Erkenntnissen über Reformprozesse zu gelangen. Denn nur durch die Formulierung von ökonomischen Modellen, welche über die wohlfahrtstheoretische Perspektive hinausgehen, wird es gelingen, eine hinreichend verlässliche Abbildung der Realität zu leisten. Hierzu bedarf es aber insbesondere einer besseren psychologischen Fundierung der tatsächlichen Handlungsmotive wirtschaftlicher und politischer Akteure, um geeignete wirtschaftspolitische Schlussfolgerungen zu formulieren.

\section{FIDE XXIII Congress}

Herausgegeben von Heribert Franz Koeck und Margit Maria Karollus
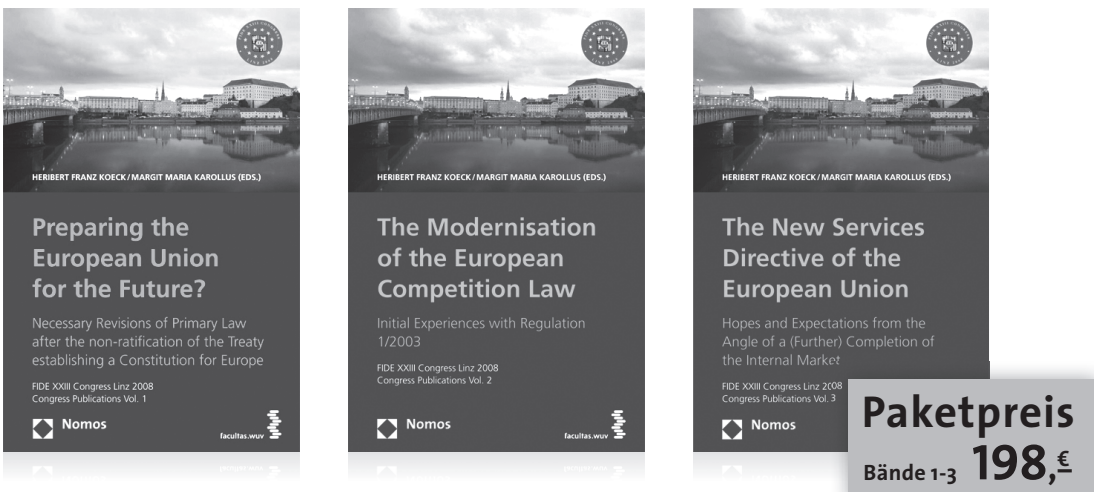

Preparing the European Union for the Future?

Congress Publications Vol. 1

2008, 410 S., brosch., 79,- $€$, ISBN 978-3-8329-3344-9

\section{The Modernisation of the}

\section{European Competition Law}

Congress Publications Vol. 2

2008, 521 S., brosch., 79,- $€$,

ISBN 978-3-8329-3345-6

\section{The New Services Directive of the European Union \\ Congress Publications Vol. 3 \\ 2008, 428 S., brosch., 79,- $€$, ISBN 978-3-8329-3346-3}

\section{TIPP}

\section{Band 1-3}

Congress Publications Vol. 1-3

2008, 3 Bände, 1.421 S., brosch., 198,- $€$, ISBN 978-3-8329-3347-O 